\title{
Longitudinal analysis of cardiac structure and function in incident-automated peritoneal dialysis: comparison between icodextrin solution and glucose-based solution
}

Jin-Bor Chen ${ }^{1 *}\left(\mathbb{D}\right.$, Ben-Chung Cheng ${ }^{1}$, Wen-Hao Liư², Shang-Chih Liao ${ }^{3}$, Mao-Young Morgan Fu², Sin-Hua Moi ${ }^{4}$ and Cheng-Hong Yang ${ }^{4}$

\begin{abstract}
Background: This study aimed to evaluate the longitudinal changes in cardiac structure and function in incidentautomated peritoneal dialysis (APD) patients.

Methods: We conducted a 2-year prospective, randomized, open-label, parallel-group study to compare the efficacy of icodextrin solution versus glucose-based solution. Echocardiography was performed at baseline, 1 and 2 years. Echocardiographic parameters over 2 years were evaluated for each group, using the Friedman test. Generalized linear regression analysis was used to test the associations between baseline clinical variables and echocardiographic changes, and a multivariate model was used to analyze cardiac function between the two groups.

Results: A total of 43 APD patients were enrolled in the beginning of this study. Twenty patients in the icodextrin group (ICO) and 18 patients in the glucose group (GLU) completed the study. In left ventricular (LV) systolic function measurements, ejection fraction (EF) increased significantly in the GLU group. Measurements of LV diastolic function and septal early mitral annulus velocity (EMV) increased significantly from baseline to 24-months in the ICO group (5. 43-5.51 ms). The GLU group showed a significant decrease in peak early diastolic velocity (EDV) (70.67-68.25 cm/s), but a significant increase in septal EMV (5.94-7.57 ms) from baseline to 24-months. No significant association was found between the baseline clinical variables and echocardiographic changes within 24 months in the generalized linear regression analysis. Multivariate models were used to investigate changes in the four primary endpoints, namely, myocardial performance index (MPI), left ventricular ejection fraction (LVEF), deceleration time (DT), and E/e' ratio. These primary endpoints show no significant association with the baseline values in both the ICO and GLU groups.
\end{abstract}

Conclusion: The present study demonstrates that long-dwell icodextrin solution can maintain reasonable cardiac structure and function in incident-APD patients.

Trial registration: ISRCTN14931270 (retrospectively registered on 23/03/2018).

Keywords: Icodextrin solution, Glucose-based solution, Echocardiogram, Automated peritoneal dialysis

\footnotetext{
* Correspondence: chenjb1019@gmail.com

${ }^{1}$ Division of Nephrology, Department of Internal Medicine, Kaohsiung Chang Gung Memorial Hospital, Chang Gung University College of Medicine, 123 Ta Pei Rd, Niao Song District, Kaohsiung, Taiwan

Full list of author information is available at the end of the article
}

(c) The Author(s). 2018 Open Access This article is distributed under the terms of the Creative Commons Attribution 4.0 International License (http://creativecommons.org/licenses/by/4.0/), which permits unrestricted use, distribution, and reproduction in any medium, provided you give appropriate credit to the original author(s) and the source, provide a link to the Creative Commons license, and indicate if changes were made. The Creative Commons Public Domain Dedication waiver (http://creativecommons.org/publicdomain/zero/1.0/) applies to the data made available in this article, unless otherwise stated. 


\section{Background}

Cardiovascular disease has been recognized as the leading cause of mortality in patients with chronic kidney disease (CKD) [1]. Among the clinical manifestations of patients with $C K D$, heart failure is the most prevalent. Clinical presentation is commonly preceded by structural cardiac disease, evidenced by image examinations $[2,3]$. A prior study revealed that CKD patients exhibited increased left ventricular (LV) mass index, left atrial volume index, and diastolic dysfunction, with their status deteriorating with the progression of CKD [4]. Another study investigated cardiac function in predialysis CKD patients, and revealed diastolic dysfunction indicated by an increase in the ratio of mitral inflow velocity and mitral annulus velocity, and left atrial volume indexed for height, prior to dialysis initiation [3]. In addition, a previous study demonstrated that, among prevalent peritoneal dialysis (PD) patients, those without a history of heart failure had an increased ratio of mitral inflow velocity and mitral annulus velocity, and that deceleration time indicated diastolic dysfunction [5]. Thus, evaluation of cardiac structure and function in CKD patients is crucial in long-term CKD management.

Icodextrin (ICO) is a glucose polymer with an average molecular weight of $17,000 \mathrm{Da}$. It can act as a colloid osmotic agent in PD therapy. ICO solution has provided advantages over glucose-based PD solution for longdwell exchange with sustained net ultrafiltration to $16 \mathrm{~h}$ on continuous ambulatory peritoneal dialysis and APD, with less damage to the peritoneal membrane [6]. In one previous study with high transport diabetic PD patients, the ICO solution demonstrated advantages in peritoneal ultrafiltration and fluid control, and facilitated metabolic control compared with the glucose-based solution in a 12-month study period [7]. Moreover, one study assessed the ultrafiltration effect of ICO solution in the nocturnal schedule of APD, showing that the ICO solution might allow sustained ultrafiltration and sodium removal [8]. Regarding the association between ICO use and echocardiographic parameters in PD patients, a study revealed significantly decreased left ventricular mass and extracellular water over a 4-month observation period [9]. Nevertheless, more detailed echocardiographic parameters are not available in the literature. Thus, a well-designed prospective study is needed to address the effect of ICO solution on cardiac structure and function in PD patients.

We hypothesized that the use of ICO in PD therapy has an advantage in cardiac function via sustained ultrafiltration compared to GLU-based solution. In the present study, we aimed to compare the impact of ICO- and GLU-based solutions on cardiac structure and function in a 2-year longitudinal period in incident-APD patients. A prospective randomized study was conducted in a cohort of APD patients who were undergoing a newly initiated
APD therapy with long-dwell exchange in the daytime. A series of echocardiographies were performed to assess cardiac structure and function during the study period.

\section{Methods}

This prospective, randomized, open-level, parallel-group study was started in June 2009 and was completed in May 2015. All of the participants were selected from the PD unit in Kaohsiung Chang Gung Memorial Hospital in Taiwan. The main inclusion criterion was adult incident-PD patients who agreed to receive nocturnal APD regimen with daily dwell. We used a purposive sampling method to enroll study participants in the outpatient department. After the study protocol was explained and informed consent was obtained, we used a computer-generated block randomization method to categorize enrolled participants into two groups. All of the participants underwent nocturnal APD with varying concentrations of glucose-based PD solutions (1.36, 2.27, and 3.86\%; Baxter Healthcare SA, Singapore) depending on the prescription from their respective nephrologists. In the ICO group, the participants received long-dwell exchange for 10-12 $\mathrm{h}$ with a $7.5 \%$ ICO PD solution (Baxter Healthcare SA, Singapore) in the daytime. In the GLU group, the participants received 1 or 2 exchanges with the glucose-based PD solution in the daytime. Cardiac structure and function were examined on echocardiography at baseline and subsequently in 1-year intervals. The study duration was 2 years.

The inclusion criteria for the study were age older than 18 years, ability to provide written informed consent, and new incident stage $5 \mathrm{CKD}$ patients who agreed to receive renal replacement therapy with the APD regimen and tolerate a long-dwell time of $\geq 10 \mathrm{~h}$ with $7.5 \%$ ICO solution. The exclusion criteria were starch allergy, glycogen storage disease, a life expectancy of $<12$ months, serious disease within 30 days before randomization, participation in another interventional study, pregnancy or lactation, or a significant psychiatric disorder that would interfere with their ability to provide informed consent and/or comply with the study procedures. Finally, 43 patients who met the inclusion criteria were enrolled, and 38 patients completed the study (Fig. 1).

During the study period, the patients were requested to visit the PD outpatient clinic at least once every month, and were examined by PD nurses using the PD protocol through telephone contact at least once every week. Data were collected from the PD home record, including body weight, blood pressure level, ultrafiltration (UF), amount, and concentration of the PD solution used. Laboratory data, including hemogram and biochemistry results, were examined at baseline and monthly thereafter. Blood levels of intact parathyroid hormone (iPTH), high-sensitivity Creactive protein (hsCRP), and glycohemoglobin (HbA1c) 


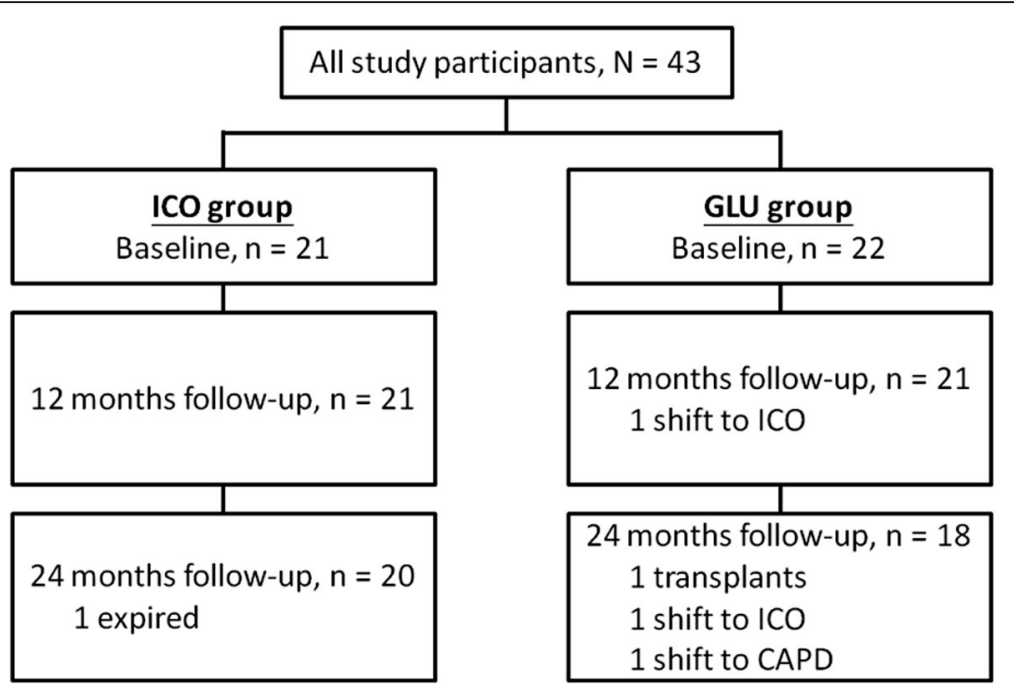

Fig. 1 Flowchart of patient selection and follow-up status

were measured at baseline and every 6 months. All blood samples were measured by using commercial kits and an auto-analyzer (Hitachi 7600-210, Hitachi Ltd., Tokyo, Japan). Albumin levels were measured using the bromocresol green method.

Standard peritoneal equilibration test (PET) was performed 1 month after APD commencement. The procedure used a 4 -h dwell for a $2.27 \%$ glucose-containing PD solution. Twenty-four urine and dialysate samples were collected for measurement of residual glomerular filtration rate, and total weekly urea and creatinine clearance. Thereafter, PET was performed every 6 months within the study period.

Echocardiographic examinations (two-dimensional and M-mode) were performed in all participants by an experienced cardiologist using an ultrasonography system (model 260 SS-A equipped with a $2.5-\mathrm{MHz}$ phased array transducer, Toshiba, Tokyo, Japan). All of the patient examinations were performed in the left lateral recumbent position. All echocardiographic data were evaluated according to the guidelines of the American Society of Echocardiography.

Cardiac performance was evaluated using pulsed Doppler echocardiography, which was conducted at the left ventricular inflow tract under two-dimensional guidance in the apical four-chamber view. The sample volume was positioned at the tips of the mitral valve leaflets and aligned to make the angle between the ultrasound beam and the blood flow vector, as determined based on color flow images, as close to zero as possible. Echocardiographic data were evaluated for cardiac structure (left atrial diameter [LAD], left ventricular end-diastolic dimension [LVEDD], left ventricular end-systolic dimension [LVESD], left ventricular end-diastolic volume [LVEDV], left ventricular endsystolic volume [LVESV], interventricular septal thickness
[IVS]), left ventricular systolic function (myocardial performance index [MPI], and left ventricular ejection fraction [LVEF]), and left ventricular diastolic function (deceleration time [DT], peak early diastolic velocity, and septal early mitral annulus velocity). LVEF and MPI were calculated using standard formulas and data obtained from echocardiography. $E / e^{\prime}$ indicated left ventricular diastolic function, and was calculated by dividing the peak early diastolic velocity by the septal early mitral annulus velocity.

After commencement of the study, patient medications, including antihypertensives, were adjusted according to clinical need. PD glucose concentration and fill volume were also adjusted as necessary. The study protocol was approved by the Committee on Human Research at Kaohsiung Chang Gung Memorial Hospital (98-0390B). The study was conducted in accordance with the Declaration of Helsinki.

The baseline characteristics of the participants were summarized and presented as mean \pm standard deviation (SD), median and interquartile range, frequency, and percentage. The differences in baseline characteristics between the two groups were estimated by using the $\chi^{2}$ test and independent two-sample $t$ test. Echocardiographic parameters from the baseline to 12 and 24 months of observation were evaluated for each group using the Friedman test. Associations between baseline variables and echocardiographic changes in the study period were analyzed by using a generalized linear regression model. Multivariate linear regression analysis was used to evaluate the 24-month changes in the four primary end points(MPI, LVEF, DT, and $\left.E / e^{\prime}\right)$.

\section{Results}

At the beginning of the study, 43 participants were enrolled - 21 participants in the ICO group, and 22 
participants in the GLU group. Twenty participants in the ICO group, and 18 participants in the GLU group completed the study (Fig. 1). Males were predominant in the ICO group; and females were predominant in the GLU group. Diabetic nephropathy was more prevalent in the ICO group. Baseline levels of $\mathrm{Hb}, \mathrm{Cr}$, cholesterol, daily ultrafiltration amount and weekly renal $\mathrm{Kt} / \mathrm{V}$ significantly differed between the two groups (Table 1).

Compared with the ICO group, the GLU group showed significantly lower baseline LVESD $(35.00 \mathrm{~mm}$

Table 1 Baseline laboratory and peritoneal equilibration test data

\begin{tabular}{|c|c|c|c|c|c|}
\hline \multirow[t]{2}{*}{ Variable } & \multicolumn{2}{|c|}{ Icodextrin $(n=21)$} & \multicolumn{2}{|c|}{ Glucose $(n=22)$} & \multirow[t]{2}{*}{$P$} \\
\hline & Mean & SD & Mean & SD & \\
\hline Age (years) & 44.37 & 12.59 & 48.22 & 12.87 & 0.327 \\
\hline Sex & & & & & $<0.001$ \\
\hline Male (n, \%) & 15 & 71.43 & 5 & 22.73 & \\
\hline Female (n, \%) & 6 & 28.57 & 17 & 77.27 & \\
\hline DM nephropathy (n, \%) & 6 & 28.57 & 1 & 4.55 & 0.046 \\
\hline Antihypertensive (n, \%) & 16 & 76.19 & 14 & 63.64 & 0.241 \\
\hline ACEi/ARB $(n, \%)$ & 15 & 71.43 & 10 & 45.45 & 0.051 \\
\hline PET & & & & & 0.077 \\
\hline High (n, \%) & 5 & 23.81 & 0 & - & \\
\hline High average (n, \%) & 9 & 42.86 & 9 & 40.91 & \\
\hline Low average (n, \%) & 5 & 23.81 & 10 & 45.45 & \\
\hline Low (n, \%) & 2 & 9.52 & 3 & 13.64 & \\
\hline $\mathrm{SBP}(\mathrm{mm} \mathrm{Hg})$ & 138.38 & 20.31 & 131.41 & 19.75 & 0.261 \\
\hline $\mathrm{DBP}(\mathrm{mm} \mathrm{Hg})$ & 85.10 & 9.86 & 85.91 & 12.66 & 0.816 \\
\hline UF amount ${ }^{\mathrm{a}}(\mathrm{ml} /$ day $)$ & 1100 & $(663-1400)$ & 775 & $(600-900)$ & 0.021 \\
\hline $\mathrm{BMI}$ & 23.73 & 3.26 & 22.61 & 4.90 & 0.387 \\
\hline $\mathrm{HbA1C}(\%)$ & 5.68 & 1.01 & 5.47 & 0.89 & 0.486 \\
\hline $\mathrm{Hb}(\mathrm{g} / \mathrm{dL})$ & 9.70 & 1.45 & 11.13 & 1.58 & 0.004 \\
\hline Hct (\%) & 29.23 & 5.43 & 34.05 & 4.67 & 0.003 \\
\hline Albumin (g/dL) & 3.65 & 0.39 & 3.66 & 0.42 & 0.954 \\
\hline BUN (mg/dL) & 59.33 & 15.93 & 59.09 & 21.03 & 0.966 \\
\hline $\mathrm{Cr}(\mathrm{mg} / \mathrm{dL})$ & 11.32 & 2.00 & 9.55 & 2.65 & 0.018 \\
\hline Sugar $(\mathrm{mg} / \mathrm{dL})^{\mathrm{a}}$ & 98 & $(84-106)$ & 94.5 & $(89-103)$ & 0.455 \\
\hline Calcium (mg/dL) & 9.06 & 1.04 & 8.83 & 0.59 & 0.374 \\
\hline$P(\mathrm{mg} / \mathrm{dL})$ & 4.97 & 1.51 & 4.82 & 1.17 & 0.719 \\
\hline $\mathrm{Na}(\mathrm{meq} / \mathrm{L})$ & 132.00 & 17.34 & 139.18 & 3.39 & 0.064 \\
\hline $\mathrm{K}(\mathrm{meq} / \mathrm{L})$ & 3.94 & 0.65 & 3.94 & 0.43 & 0.992 \\
\hline i-PTH $(p g / m L)^{a}$ & 151 & $(93.7-286)$ & 328.5 & $(113-602)$ & 0.991 \\
\hline Cholesterol (mg/dL) & 166 & (130-198) & 210.5 & $(180-241)$ & 0.001 \\
\hline Triglyceride $(\mathrm{mg} / \mathrm{dL})^{\mathrm{a}}$ & 135 & $(78-178)$ & 89 & $(72-178)$ & 0.371 \\
\hline hsCRP (mg/L) & 3.91 & 4.03 & 6.04 & 9.61 & 0.353 \\
\hline Renal Kt/N weekly & 0.45 & 0.27 & 0.74 & 0.41 & 0.011 \\
\hline Total Kt $/ N$ weekly & 2.04 & 0.36 & 2.18 & 0.46 & 0.264 \\
\hline Renal Ccr weekly & 22.66 & 14.33 & 29.69 & 18.40 & 0.171 \\
\hline Total Ccr weekly & 62.90 & 13.95 & 64.92 & 16.83 & 0.671 \\
\hline PGLI & 0.31 & 0.09 & 0.32 & 0.16 & 0.768 \\
\hline
\end{tabular}

$P$ value is estimated from the Fisher's exact, $X^{2}$ or independent two-sample $t$-test

Abbreviations: $A C E i$ angiotensin converting enzyme inhibitor, $A R B$ angiotensin receptor blocker, SBP systolic blood pressure, $D B P$ diastolic blood pressure, UF ultrafiltration, $B M I$ body mass index, $h s C R P$ high-resolution $C$-reactive protein, $P G L I$ peritoneal glucose loading index

${ }^{a}$ Data are presented as median (interquartile range) 
vs $30.49 \mathrm{~mm})$, and LVESV values $\left(53.48 \mathrm{~mm}^{3}\right.$ vs 39 . $00 \mathrm{~mm}^{3}$ ) (Table 2).

Cardiac structure measurements, including LAD, LVEDD, LVESD, LVEDV, LVESV, and IVS, in both groups were significantly altered from baseline to 24 months. In the ICO group, LAD significantly increased, while LVEDV, LVESV, IVS significantly decreased. In the GLU group, LAD, LVEDD, LVESD, LVEDV, and LVESV all significantly increased. In LV systolic function measurement, only LVEF had a significant increase in the GLU group. In the measurements of LV diastolic function, only septal EMV showed significant increase from baseline to 24 months in the ICO group (5.43-5.51 ms). The GLU group showed a significant decrease in peak EDV $(70.67-68.25 \mathrm{~cm} / \mathrm{s})$, but a significant increase in septal EMV (5.94-7.57 ms) from baseline to 24 months (Table 3).

Table 4 presents the results of the generalized linear regression model for the association of clinical variables at baseline with changes in echocardiographic parameters at 24 months from baseline. No significant model was found in the present study. The multivariate models used to investigate changes in the four primary end points (MPI, LVEF, DT, and $E / e^{\prime}$ ratio) are presented in Table 5. The primary end points show no significant association with the baseline values in both the ICO and GLU groups.

\section{Discussion}

In the present study, we evaluated the effects of the ICO solution dwell on cardiac structure and function of incident-APD patients by performing serial echocardiographic examinations for 2 years. Overall, most echocardiographic parameters of cardiac structure showed a significant change within the 2-year period in both ICO and GLU groups. Other echocardiographic parameters of LV systolic function and diastolic function showed varying significant changes in the ICO and GLU groups. Echocardiography has been recognized as a valuable and noninvasive tool to evaluate cardiac structure and function in CKD patients. It provides information not only on measurements of ventricular mass and volume status, either in systolic or diastolic phase, but also on geometry, ejection fraction, filling pressure, and valvular disease. The National Kidney Foundation's Kidney Disease Outcomes Quality Initiative clinical practice guidelines for cardiovascular disease in dialysis patients recommends that echocardiography be performed in all patients (pediatric and adult) at the initiation of dialysis, once the patients have achieved dry weight (ideally

Table 2 Baseline echocardiographic parameters

\begin{tabular}{|c|c|c|c|c|c|c|c|}
\hline \multirow[t]{2}{*}{ Variable } & \multicolumn{3}{|c|}{ Icodextrin } & \multicolumn{3}{|c|}{ Glucose } & \multirow[t]{2}{*}{$P$} \\
\hline & $n$ & Mean & SD & $n$ & Mean & SD & \\
\hline \multicolumn{8}{|l|}{ Cardiac structure } \\
\hline LA diameter (mm) & 21 & 34.97 & 7.51 & 22 & 32.25 & 5.88 & 0.193 \\
\hline LVEDD (mm) & 21 & 50.80 & 7.66 & 22 & 46.88 & 6.36 & 0.075 \\
\hline LVESD (mm) & 21 & 35.00 & 6.35 & 22 & 30.49 & 6.14 & 0.023 \\
\hline LVEDV $\left(\mathrm{mm}^{3}\right)$ & 21 & 124.71 & 42.49 & 22 & 103.95 & 34.34 & 0.085 \\
\hline $\operatorname{LVESV}\left(\mathrm{mm}^{3}\right)$ & 21 & 53.48 & 21.83 & 22 & 39.00 & 22.22 & 0.037 \\
\hline IVS (mm) & 21 & 12.65 & 2.29 & 22 & 11.53 & 1.79 & 0.082 \\
\hline \multicolumn{8}{|l|}{ LV systolic function } \\
\hline MPI & 21 & 0.37 & 0.19 & 22 & 0.49 & 0.57 & 0.367 \\
\hline LVEF (\%) & 21 & 57.81 & 10.03 & 22 & 63.40 & 9.51 & 0.068 \\
\hline \multicolumn{8}{|l|}{ LV diastolic function } \\
\hline Deceleration time (ms) & 21 & 169.81 & 73.50 & 22 & 213.59 & 85.21 & 0.079 \\
\hline Peak early diastolic velocity $(\mathrm{cm} / \mathrm{s})$ & 20 & 71.89 & 34.66 & 22 & 70.67 & 28.75 & 0.902 \\
\hline Septal EM velocity (ms) & 21 & 5.43 & 3.00 & 22 & 5.94 & 1.58 & 0.482 \\
\hline$E / e^{\prime}$ & 20 & 11.70 & 6.36 & 22 & 12.38 & 5.24 & 0.707 \\
\hline \multicolumn{8}{|l|}{ RV function } \\
\hline TAPSE & 21 & 20.96 & 3.28 & 22 & 21.07 & 2.64 & 0.900 \\
\hline PAsP & 21 & 20.65 & 9.70 & 22 & 18.81 & 4.82 & 0.409 \\
\hline
\end{tabular}

$P$ value was estimated from the independent two-sample $t$ test

Abbreviations: LA left atrium, LVEDD left ventricular end-diastolic dimension, LVESD left ventricular end-systolic dimension, LVEDV left ventricular end-diastolic volume, LVESV left ventricular end-systolic volume, LVEF left ventricular ejection fraction, IVS interventricular septum, MPI myocardial performance index, EM early mitral annulus, E/e' peak early diastolic velocity/septal EM velocity, RVright ventricle, TAPSE tricuspid annular plane systolic excursion, PAsP peak systolic pulmonary pressure 
Table 3 The series of echocardiographic parameters among the icodextrin and glucose users

\begin{tabular}{|c|c|c|c|c|c|c|}
\hline & \multicolumn{6}{|c|}{ Cardiac structure } \\
\hline & $\mathrm{LAD}(\mathrm{mm})$ & LVEDD (mm) & $\operatorname{LVESD}(\mathrm{mm})$ & LVEDV $\left(\mathrm{mm}^{3}\right)$ & LVESV $\left(\mathrm{mm}^{3}\right)$ & IVS (mm) \\
\hline \multicolumn{7}{|l|}{ Icodextrin } \\
\hline Baseline $(n=21)$ & $34.97 \pm 7.51$ & $50.80 \pm 7.66$ & $35.00 \pm 6.35$ & $124.71 \pm 42.49$ & $53.48 \pm 21.83$ & $12.65 \pm 2.29$ \\
\hline After 12 months $(n=21)$ & $36.90 \pm 61.16$ & $50.48 \pm 7.93$ & $34.90 \pm 6.86$ & $127.76 \pm 40.22$ & $50.24 \pm 19.79$ & $12.76 \pm 2.10$ \\
\hline After 24 months $(n=20)$ & $35.90 \pm 10.99$ & $50.85 \pm 14.59$ & $32.05 \pm 10.46$ & $121.35 \pm 59.03$ & $46.20 \pm 31.98$ & $11.45 \pm 4.48$ \\
\hline$P$ - value & 0.001 & 0.195 & 0.063 & 0.003 & 0.004 & 0.004 \\
\hline \multicolumn{7}{|l|}{ Glucose } \\
\hline Baseline $(n=22)$ & $32.25 \pm 5.88$ & $46.88 \pm 6.36$ & $30.49 \pm 6.14$ & $103.95 \pm 34.34$ & $39.00 \pm 22.22$ & $11.53 \pm 1.79$ \\
\hline After 12 months $(n=21)$ & $33.90 \pm 5.08$ & $47.24 \pm 7.50$ & $31.52 \pm 6.61$ & $106.43 \pm 40.43$ & $42.52 \pm 23.15$ & $11.33 \pm 1.91$ \\
\hline After 24 months $(n=18)$ & $34.83 \pm 5.45$ & $49.22 \pm 8.43$ & $31.67 \pm 7.47$ & $118.39 \pm 47.99$ & $43.67 \pm 27.06$ & $11.33 \pm 1.78$ \\
\hline \multirow[t]{3}{*}{$P$ - value } & 0.005 & 0.001 & 0.001 & $<0.001$ & 0.001 & 0.005 \\
\hline & \multicolumn{2}{|c|}{ LV systolic function } & \multicolumn{4}{|c|}{ LV diastolic function } \\
\hline & MPI & LVEF (\%) & DT (ms) & Peak EDV (cm/s) & Septal EMV (ms) & $E / e^{\prime}$ \\
\hline \multicolumn{7}{|l|}{ Icodextrin } \\
\hline Baseline $(n=21)$ & $0.37 \pm 0.19$ & $57.81 \pm 10.03$ & $169.81 \pm 73.50$ & $71.89 \pm 34.66$ & $5.43 \pm 3.00$ & $11.70 \pm 6.36$ \\
\hline After 12 months $(n=21)$ & $0.43 \pm 0.20$ & $60.43 \pm 7.29$ & $214.24 \pm 72.01$ & $73.89 \pm 27.94$ & $6.05 \pm 2.42$ & $12.83 \pm 6.60$ \\
\hline After 24 months $(n=20)$ & $0.32 \pm 0.21$ & $56.55 \pm 21.88$ & $193.35 \pm 75.03$ & $65.80 \pm 33.68$ & $5.51 \pm 2.28$ & $11.51 \pm 9.60$ \\
\hline$P$ - value & 0.195 & 0.068 & 0.081 & 0.150 & 0.035 & 0.104 \\
\hline \multicolumn{7}{|l|}{ Glucose } \\
\hline Baseline $(n=22)$ & $0.49 \pm 0.57$ & $63.40 \pm 9.51$ & $213.60 \pm 85.21$ & $70.67 \pm 28.75$ & $5.94 \pm 1.58$ & $12.38 \pm 5.24$ \\
\hline After 12 months $(n=21)$ & $0.35 \pm 0.18$ & $60.67 \pm 8.50$ & $199.62 \pm 79.76$ & $66.52 \pm 23.69$ & $5.97 \pm 3.11$ & $10.46 \pm 5.05$ \\
\hline After 24 months $(n=18)$ & $0.30 \pm 0.19$ & $64.50 \pm 8.58$ & $201.94 \pm 62.55$ & $68.25 \pm 20.31$ & $7.57 \pm 3.42$ & $9.08 \pm 4.35$ \\
\hline$P$ - value & 0.174 & 0.015 & 0.051 & 0.036 & 0.013 & 0.052 \\
\hline
\end{tabular}

$P$ value was estimated from Friedman test

Abbreviation: $D T$ deceleration time

within 1-3 months of initiation) and at 3-year intervals [10]. Several clinical studies have been conducted on the use of serial echocardiographies to evaluate cardiac structure and function in varying stages of CKD. In the subset patient analysis in a chronic renal insufficiency cohort study, results demonstrated that mean left ventricular mass index did not change from progression to advanced CKD to dialysis therapy initiation. However, ejection fraction declined during this transition period [11]. In the IDEAL (Initiating Dialysis Early and Late) trial, the investigators did not find any changes in echocardiographic parameters 12 months after dialysis initiation [3]. In the present study, we found that, in both groups, most of the echocardiographic parameters significantly differed in the 2 -year study period. The above-mentioned observations imply that abnormalities in the cardiac structure and function of CKD patients resulted from uremic milieu, the progression of which could not be inhibited by dialysis initiation [12]. However, the echocardiographic results provide valuable information to clinicians in clinical practice. Based on this, a suitable therapeutic strategy combining dialysis regimen administration, drug adjustment, and lifestyle modification can be planned.

LAD has been hypothesized as an indicator of the integration of LV diastolic performance over time. Thus, left atrium (LA) volume provides a prospective view of diastolic dysfunction [13, 14]. Moreover, LA size is one of the most important echocardiographic risk predictors of LV hypertrophy. Prior studies have demonstrated the correlation between LA size and LV mass [14-16]. In the present study, results showed a LAD increment at 12 months in the ICO group and at 24 months in the GLU group. LAD is not reduced with either the ICO solution or the GLU solution over 24 months. Our result contradicts that of the study by Io et al., as they observed a reduction in LA size in PD patients after 24 months [14]. The exact explanation is not clear; however, the hemoglobin level in the Io study was indicative of a more severe anemia than that in the present study. We speculate that anemia management may be a contributing factor in LA size reduction in 24 months. However, the small sample size in both studies might have biased the results. 
Table 4 Generalized linear regression analysis of the association between baseline clinical variables and changes in the echocardiographic parameters from baseline to 24 months

\begin{tabular}{|c|c|c|c|c|}
\hline Dependent variable & RMSE & $R^{2}$ & $\mathrm{~F}$ & $P$ \\
\hline \multicolumn{5}{|l|}{ Cardiac structure } \\
\hline LA diameter (mm) & 7.43 & 0.88 & 0.75 & 0.716 \\
\hline LVEDD (mm) & 15.00 & 0.89 & 0.82 & 0.680 \\
\hline LVESD (mm) & 7.75 & 0.94 & 1.59 & 0.398 \\
\hline LVEDV $\left(\mathrm{mm}^{3}\right)$ & 62.12 & 0.88 & 0.70 & 0.745 \\
\hline $\operatorname{LVESV}\left(\mathrm{mm}^{3}\right)$ & 14.64 & 0.97 & 3.44 & 0.168 \\
\hline IVS (mm) & 2.74 & 0.94 & 1.61 & 0.392 \\
\hline \multicolumn{5}{|l|}{ LV systolic function } \\
\hline MPI & 0.25 & 0.98 & 4.37 & 0.125 \\
\hline LVEF (\%) & 22.33 & 0.87 & 0.65 & 0.778 \\
\hline \multicolumn{5}{|l|}{ LV diastolic function } \\
\hline Deceleration time (ms) & 54.74 & 0.96 & 2.42 & 0.256 \\
\hline Peak early diastolic velocity $(\mathrm{cm} / \mathrm{s})$ & 44.19 & 0.87 & 0.66 & 0.768 \\
\hline Septal EM velocity (ms) & 4.77 & 0.80 & 0.41 & 0.918 \\
\hline$E / e^{\prime}$ & 12.68 & 0.79 & 0.38 & 0.932 \\
\hline
\end{tabular}

All sociodemographic variables, PET variables, and icodextrin/glucose were included as covariates in the model

Congestive heart failure (CHF) is a common presenting symptom of cardiovascular disease in the dialysis population [17-21]. An earlier study demonstrated that the prevalence of $\mathrm{CHF}$ from the start of dialysis therapy and its subsequent annual incidence were high [18]. In the present study, CHF was not evidenced at baseline by indicators of LV systolic function (MPI and LVEF) and diastolic function (DT and $\left.E / e^{\prime}\right)$. In the study by Wang, et al., elevated $E / e^{\prime}$ ratio ( $>15$ ) was found in more than $50 \%$ of the prevalent PD patients [22]. E/e' ratio was an independent risk factor for all-cause mortality and cardiovascular mortality [22]. The present study showed that the $E / e^{\prime}$ ratio was progressively reduced after
24 months in the incident-APD patients, either in the ICO or GLU dwell. Nevertheless, other indicators of LV diastolic function in both groups did not change markedly at 24 months. The results indicate that ICO and GLU dwells in incident-APD patients maintain reasonable cardiac function over 24 months.

Residual renal function (RRF) not only provides a small solute clearance, but also maintains fluid control in PD patients. RRF supports the maintenance of cardiovascular health of PD patients [23]. RRF was significantly associated with LVH, independent of hypertension, anemia, and hypoalbuminemia [24]. In the present study, parameters of cardiac structure and cardiac function

Table 5 Multivariate model analysis for the primary end points at 24 months

\begin{tabular}{|c|c|c|c|c|c|c|c|c|}
\hline End Point & Parameter & Estimate & SE & $t$ & $P$ & $\mathrm{n}$ & $R^{2}$ & Adjusted $R^{2}$ \\
\hline \multirow[t]{3}{*}{ MPI } & Intercept & 0.03 & 0.08 & 0.41 & 0.682 & 38 & 0.01 & -0.05 \\
\hline & Baseline MPI & -0.02 & 0.07 & -0.34 & 0.733 & & & \\
\hline & Icodextrin (vs. glucose) & 0.31 & 0.05 & 5.60 & 0.000 & & & \\
\hline \multirow[t]{3}{*}{ LVEF } & Intercept & 0.33 & 0.30 & 1.10 & 0.280 & 38 & 0.09 & 0.03 \\
\hline & Baseline LVEF & 6.60 & 5.63 & 1.17 & 0.249 & & & \\
\hline & Icodextrin (vs. glucose) & 37.01 & 18.20 & 2.03 & 0.050 & & & \\
\hline \multirow[t]{3}{*}{ DT } & Intercept & 0.23 & 0.20 & 1.16 & 0.253 & 38 & 0.04 & -0.01 \\
\hline & Baseline DT & 3.92 & 22.80 & 0.17 & 0.864 & & & \\
\hline & Icodextrin (vs. glucose) & 152.00 & 38.78 & 3.92 & 0.000 & & & \\
\hline \multirow[t]{3}{*}{$E / e^{\prime}$} & Intercept & 0.32 & 0.27 & 1.18 & 0.248 & 35 & 0.06 & 0.00 \\
\hline & Baseline E/e' & -1.74 & 2.65 & -0.66 & 0.517 & & & \\
\hline & Icodextrin (vs. glucose) & 7.33 & 3.75 & 1.95 & 0.060 & & & \\
\hline
\end{tabular}


were not associated with RRF, indicated by weekly renal creatinine clearance in the generalized liner regression analysis. We expect future studies to clarify the association between RRF and whole cardiac function.

The ICO solution was characterized by a longer dwell time than that with the GLU solution. A meta-analysis of ICO solution in PD therapy demonstrated that ICO solution provided greater fluid removal and small solute clearance [6]. In the present study, we intended to investigate the association between changes in cardiac structure and function in ICO dwell in incident-APD patients. In the multivariate analysis, we did not find any advantage of the ICO dwell when compared with the GLU dwell in terms of the parameters of cardiac systolic (MPI and LVEF) and diastolic functions (DT and E/e') for a 24-month period in incident-APD patients. Nevertheless, the ICO dwell maintains LV systolic and diastolic functions over 24 months. This result indicates that a long dwell time with ICO solution in daytime is not harmful to the cardiac function of APD patients.

Although the present study had a prospective randomized design, it still has some limitations. First, the participants had more favorable baseline characteristics, including age, blood pressure levels, and cardiac function. These characteristics may mask significant changes in echocardiographic parameters after PD initiation. Second, the sample size was relatively small and might have affected the statistical power of our analyses. Furthermore, cardiac structure and LV systolic function (LVEF) at baseline worsened more in the ICO solution group than in the GLU solution group, and might have masked the advantage of the ICO dwell over the GLU dwell in the period after APD initiation.

\section{Conclusions}

The present study demonstrated that no major differences in cardiac structure and function were observed when comparing ICO and GLU solutions in incident-APD patients. Further studies, with larger sample sizes and longer study periods, are needed to evaluate the association between the ICO solution and changes in cardiac parameters by using echocardiographic examination.

\footnotetext{
Abbreviations

Ele': Peak early diastolic velocity/septal EM velocity; APD: Automated peritoneal dialysis; CHF: Congestive heart failure; CKD: Chronic kidney disease; EM: Early mitral annulus; HbA1C: Glycohemoglobin; hsCRP: Highresolution C-reactive protein; ICO: Icodextrin; IPTH: Intact parathyroid hormone; IVS: Interventricular septum; LA: Left atrium; LAD: Left atrial diameter; LV: Left ventricle; LVEDD: Left ventricular end-diastolic dimension; LVEDV: Left ventricular end-diastolic volume; LVEF: Left ventricular ejection fraction; LVESD: Left ventricular end-systolic dimension; LVESV: Left ventricular end-systolic volume; MPI: Myocardial performance index; PD: Peritoneal dialysis; PET: Peritoneal equilibration test; RRF: Residual renal function
}

\section{Acknowledgments}

The authors thank Yu-Chuan Huang and Li-Chueh Kuo for their assistance in data management.

\section{Funding}

This work was supported by Baxter-Clinical Evidence Council (CEC) Fund (08CEC2AP002). The Fund supported the fee for laboratory examinations in the study.

\section{Availability of data and materials}

All data supporting the study is presented in the manuscript or available upon request from the corresponding author of this manuscript, Jin-Bor Chen.

\section{Authors' contributions}

JBC design the study and drafted the manuscript. BCC acquisition of data and interpretation of data. WHL performed echocardiography examinations. MYMF interpreted the echocardiography results. SCL critically reviewed the clinical data and revised it critically for important intellectual content. SHM performed statistical analysis. CHY critically reviewed the statistical data and revised it critically for important intellectual content. All authors read and approved the final manuscript.

\section{Authors' information}

Jin-Bor Chen and Ben-Chung Cheng worked at the Division of Nephrology, Department of Internal Medicine, Kaohsiung Chang Gung Memorial Hospital and Chang Gung University College of Medicine, Kaohsiung, Taiwan. WenHao Liu, and Mao-Young Morgan Fu worked at the Division of Cardiology, Department of Internal Medicine, Kaohsiung Chang Gung Memorial Hospital, Chang Gung University College of Medicine, Kaohsiung, Taiwan.

Shang-Chih Liao worked at the Division of Nephrology, Kaohsiung Municipal Feng-Shan Hospital, Taiwan. Sin-Hua Moi and Cheng-Hong Yang worked at the Department of Electronic Engineering, National Kaohsiung University of Applied Sciences, Kaohsiung, Taiwan.

\section{Ethics approval and consent to participate}

The study protocol was approved by the Committee on Human Research at Kaohsiung Chang Gung Memorial Hospital (98-0390B). Informed consent obtained from participants was written. The study was conducted in accordance with the Declaration of Helsinki.

\section{Competing interests}

Jin-Bor Chen, Ben-Chung Cheng received speaker honoraria from Baxter Taiwan.

\section{Publisher's Note}

Springer Nature remains neutral with regard to jurisdictional claims in published maps and institutional affiliations.

\section{Author details}

${ }^{1}$ Division of Nephrology, Department of Internal Medicine, Kaohsiung Chang Gung Memorial Hospital, Chang Gung University College of Medicine, 123 Ta Pei Rd, Niao Song District, Kaohsiung, Taiwan. ${ }^{2}$ Division of Cardiology, Department of Internal Medicine, Kaohsiung Chang Gung Memorial Hospital, Chang Gung University College of Medicine, Kaohsiung, Taiwan. ${ }^{3}$ Division of Nephrology, Kaohsiung Municipal Feng-Shan Hospital, Kaohsiung, Taiwan.

${ }^{4}$ Department of Electronic Engineering, National Kaohsiung University of Applied Sciences, Kaohsiung, Taiwan.

Received: 11 September 2017 Accepted: 1 May 2018

Published online: 08 May 2018

References

1. Foley RN, Parfrey PS, Harnett JD, Kent GM, Martin CJ, Murray DC, Barre PE. Clinical and echocardiographic disease in patients starting end-stage renal disease therapy. Kidney Int. 1995;47:186-92.

2. Mclntyre CW. Effects of hemodialysis on cardiac function. Kidney Int. 2009; 76:371-5.

3. Whalley GA, Marwick TH, Doughty RN, Cooper BA, Johnson DW, Pilmore A, Harris DC, Pollock CA, Collins JF. Effect of early initiation of dialysis on cardiac structure and function: results from the echo substudy of the IDEAL trial. Am J Kidney Dis. 2013;61:262-70.

4. Cai QZ, Lu XZ, Lu Y, Wang AY. Longitudinal changes of cardiac structure and function in CKD (CASCADE study). J Am Soc Nephrol. 2014;25:1599-608. 
5. Wang AY, Wang M, Lam CW, Chan IH, Lui SF, Sanderson JE. Heart failure in long-term peritoneal dialysis patients: a 4-year prospective analysis. Clin J Am Soc Nephrol. 2011;6:805-12.

6. Qi H, Xu C, Yan H, Ma J. Comparison of icodextrin and glucose solutions for long dwell exchange in peritoneal dialysis: a meta-analysis of randomized controlled trials. Perit Dial Int. 2011;31:179-88.

7. Paniagua R, Ventura MD, Avila-Diaz M, Cisneros A, Vicente-Martinez M, Furlong MD, Garcia-Gonzalez Z, Villanueva D, Orihuela O, Prado-Uribe MD, et al. Icodextrin improves metabolic and fluid management in high and high-average transport diabetic patients. Perit Dial Int. 2009;29:422-32.

8. Rodriguez-Carmona A, Perez Fontan M, Garcia Lopez E, Garcia Falcon T, Diaz Cambre H. Use of icodextrin during nocturnal automated peritoneal dialysis allows sustained ultrafiltration while reducing the peritoneal glucose load: a randomized crossover study. Perit Dial Int. 2007;27:260-6.

9. Konings CJ, Kooman JP, Schonck M, Gladziwa U, Wirtz J, van den Wall Bake AW, Gerlag PG, Hoorntje SJ, Wolters J, van der Sande FM, et al. Effect of icodextrin on volume status, blood pressure and echocardiographic parameters: a randomized study. Kidney Int. 2003;63:1556-63.

10. KJDOQI Workgroup. KJDOQI clinical practice guidelines for cardiovascular disease in dialysis patients. Am J Kidney Dis. 2005;45(4 Suppl 3):S1-153.

11. Bansal N, Keane M, Delafontaine P, Dries D, Foster E, Gadegbeku CA, Go AS, Hamm LL, Kusek JW, Ojo AO, et al. A longitudinal study of left ventricular function and structure from CKD to ESRD: the CRIC study. Clin J Am Soc Nephrol. 2013;8:355-62.

12. Shroff GR, Herzog CA. Echocardiography: providing additional insights into cardiovascular structural and functional abnormalities in advanced CKD. Clin J Am Soc Nephrol. 2013;8:339-41.

13. Tsang TS, Barnes ME, Gersh BJ, Takemoto Y, Rosales AG, Bailey KR, Seward JB. Prediction of risk for first age-related cardiovascular events in an elderly population: the incremental value of echocardiography. J Am Coll Cardiol. 2003:42:1199-205.

14. Io H, Ro Y, Sekiguchi Y, Shimaoka T, Inuma J, Hotta Y, Aruga S, Inami Y, Sato $M$, Kobayashi T, et al. Cardiac function and structure in longitudinal analysis of echocardiography in peritoneal dialysis patients. Perit Dial Int. 2010;30: 353-61

15. Vaziri SM, Larson MG, Lauer MS, Benjamin EJ, Levy D. Influence of blood pressure on left atrial size. The Framingham heart study. Hypertension. 1995; 25:1155-60.

16. Gerdts E, Oikarinen L, Palmieri V, Otterstad JE, Wachtell K, Boman K, Dahlof B, Devereux RB. Correlates of left atrial size in hypertensive patients with left ventricular hypertrophy: the Losartan Intervention For Endpoint Reduction in Hypertension (LIFE) study. Hypertension. 2002;39:739-43.

17. Stack AG, Bloembergen WE. A cross-sectional study of the prevalence and clinical correlates of congestive heart failure among incident US dialysis patients. Am J Kidney Dis. 2001;38:992-1000.

18. Harnett JD, Foley RN, Kent GM, Barre PE, Murray D, Parfrey PS. Congestive heart failure in dialysis patients: prevalence, incidence, prognosis and risk factors. Kidney Int. 1995:47:884-90.

19. Cheung AK, Sarnak MJ, Yan G, Berkoben M, Heyka R, Kaufman A, Lewis J, Rocco M, Toto R, Windus D, et al. Cardiac diseases in maintenance hemodialysis patients: results of the HEMO study. Kidney Int. 2004;65:2380-9.

20. Wang AY, Woo J, Lam CW, Wang M, Sea MM, Lui SF, Li PK, Sanderson J. Is a single time point C-reactive protein predictive of outcome in peritoneal dialysis patients? J Am Soc Nephrol. 2003;14:1871-9.

21. Huting J, Schutterle G. Cardiovascular factors influencing survival in endstage renal disease treated by continuous ambulatory peritoneal dialysis. Am J Cardiol. 1992:69:123-7.

22. Wang AY, Wang M, Lam CW, Chan IH, Zhang Y, Sanderson JE. Left ventricular filling pressure by Doppler echocardiography in patients with end-stage renal disease. Hypertension. 2008;52:107-14.

23. Wang AY. The "heart" of peritoneal dialysis. Perit Dial Int. 2007;27(Suppl 2): S228-32.

24. Wang AY, Wang M, Woo J, Law MC, Chow KM, Li PK, Lui SF, Sanderson JE. A novel association between residual renal function and left ventricular hypertrophy in peritoneal dialysis patients. Kidney Int. 2002;62:639-47.

\section{Ready to submit your research? Choose BMC and benefit from:}

- fast, convenient online submission

- thorough peer review by experienced researchers in your field

- rapid publication on acceptance

- support for research data, including large and complex data types

- gold Open Access which fosters wider collaboration and increased citations

- maximum visibility for your research: over $100 \mathrm{M}$ website views per year

At BMC, research is always in progress.

Learn more biomedcentral.com/submissions 